\title{
Pallidal Stimulation for Parkinsonism: Improved Brain Activation during Sequence Learning
}

\author{
Masafumi Fukuda, MD, ${ }^{1,2}$ Maria Felice Ghilardi, MD, ${ }^{3}$ Maren Carbon, MD, ${ }^{1}$ Vijay Dhawan, PhD, ${ }^{1,2}$ \\ Yilong Ma, PhD, ${ }^{1,2}$ Andrew Feigin, MD, ${ }^{1,2}$ Marc J. Mentis, MD, ${ }^{1,2}$ Claude Ghez, MD, ${ }^{3}$ \\ and David Eidelberg, $\mathrm{MD}^{1,2}$
}

\begin{abstract}
We used ${ }^{15} \mathrm{O}$-labeled water and positron emission tomography to assess the effect of deep brain stimulation of the internal globus pallidus on motor sequence learning in Parkinson's disease. Seven right-handed patients were scanned on and off stimulation while they were performing a motor sequence learning task and a kinematically matched motor execution reference task. The scans were performed after a 12-hour medication washout. Stimulation parameters were adjusted for maximal motor improvement; experimental task parameters were held constant across stimulation conditions. Internal globus pallidus stimulation improved motor ratings by $37 \%(p<0.01)$. During the sequence learning task, stimulation improved performance as measured by several correct anticipatory movements $(p<0.01)$ and by verbal report $(p<0.001)$. Concurrent positron emission tomography imaging during learning demonstrated significant $(p<0.01)$ increases in brain activation with stimulation in the left dorsolateral prefrontal cortex, bilaterally in premotor cortex, and in posterior parietal and occipital association areas. Stimulation did not affect the activity of these regions during the performance of the motor execution reference task. These findings suggest that internal globus pallidus deep brain stimulation can enhance the activity of prefrontal cortico-striato-pallidothalamic loops and related transcortical pathways. Improved sequence learning with stimulation may be directly related to these functional changes.
\end{abstract}

Stereotaxic surgery for the medically refractory symptoms of Parkinson's disease (PD) has become increasingly accepted in clinical practice. ${ }^{1}$ In general, these interventions have focused on the motor manifestations of this illness, targeting the internal globus pallidus (GPi) and the subthalamic nucleus (STN). In contrast with ablative procedures, deep brain stimulation (DBS) of these nuclei can provide a reversible means of reducing akinesia, rigidity, and L-dopa-induced dyskinesias. ${ }^{2}$ Positron emission tomographic (PET) imaging during stimulation provides a means of studying the mechanisms of clinical benefit afforded by these interventions. Previous PET studies using ${ }^{15} \mathrm{O}$-labeled water $\left(\mathrm{H}_{2}{ }^{15} \mathrm{O}\right)$ to assess the effects of stimulation on regional brain activation during simple motor execution ${ }^{3,4}$ have supported the notion that these procedures alleviate parkinsonian symptoms by enhancing the activity of one or more nodes of the motor cortico-striatopallidothalamic circuit. $^{5}$

DBS also may affect the functioning of neural pathways relating to complex behavior. Although DBS for advanced parkinsonism generally does not alter cognitive status, ${ }^{6-8}$ specific aspects of executive function may be either improved or worsened by stimulation. ${ }^{?}$ The mechanism by which DBS might alter performance on these complex neurobehavioral tasks is unknown, although involvement of nonmotor corticostriato-pallidothalamic circuits is likely.

We have developed a series of motor learning tasks designed to evaluate the functioning of these neural pathways with imaging. ${ }^{10,11}$ In a recent study, we used PET to scan unmedicated early-stage PD patients and normal control subjects during motor sequence learning. ${ }^{12}$ Although the learning achieved during the PET
From the ${ }^{1}$ Center for Neurosciences, North Shore-Long Island Jewish Research Institute; ${ }^{2}$ Department of Neurology, North Shore University Hospital and New York University School of Medicine, Manhasset; and ${ }^{3}$ Center for Neurobiology and Behavior, Motor Control Laboratory, Columbia College of Physicians and Surgeons, New York, NY.

Received Aug 7, 2001, and in revised form Jan 17, 2002, and Mar 25. Accepted for publication Mar 27, 2002.
Address correspondence to Dr Eidelberg, Center for Neuroscience, North Shore-Long Island Jewish Research Institute, 350 Community Drive, Manhasset, NY 11030. E-mail: david1@nshs.edu 
epoch was lower in the PD patients, performance in both groups correlated significantly with the activity of a common network of brain regions comprising the dorsolateral prefrontal cortex (DLPFC), the premotor cortex (PMC), and the posterior parietal cortex. To date, however, the effects of DBS on brain activation responses during sequence learning have not been investigated.

We recently have reported the results of a psychophysical study suggesting that GPi DBS can improve motor sequence learning in more advanced PD patients. ${ }^{13}$ In this study, we used the general imaging approach described by us previously ${ }^{4}$ to determine the mechanism of this behavioral change. We scanned seven PD patients with $\mathrm{H}_{2}{ }^{15} \mathrm{O} / \mathrm{PET}$ on and off stimulation while they performed a motor sequence learning task and a kinematically matched motor execution reference task. The paired task design in conjunction with PET was used to determine whether stimulation significantly altered local activation responses during sequence learning, and whether these changes were related specifically to cognitive processing rather than to motor execution.

\section{Subjects and Methods}

We studied right-handed PD patients who underwent PET imaging during pallidal stimulation. This group consisted of seven patients (age, $51.9 \pm 12.3$ years [mean \pm standard deviation]; Hoehn and Yahr stage, $3.2 \pm 1.0$ ). They were implanted with GPi DBS for (1) bradykinesia and rigidity predominant $\mathrm{PD}$, (2) severe response fluctuations, or (3) both. Two patients underwent unilateral electrode placement in the left pallidum; the remaining five patients underwent staged bilateral surgery. Postoperative magnetic resonance imaging was used to confirm target position. The clinical characteristics of these patients and their stimula- tion parameters are presented in Table 1. Imaging data from Patients 1 to 4 have been included in previously published reports of the effects of stimulation on resting glucose utilization and brain activation responses during simple motor execution. ${ }^{4,14}$

\section{Study Design}

Patients with implanted GPi stimulators were studied over a 3-day period as described previously. ${ }^{4}$ All antiparkinsonian medications were withheld for at least 12 hours before each day of testing. The first day was utilized for task training and for the selection of experimental parameters for the PET studies. Imaging was performed over the next 2 days, with stimulation condition randomized to being on 1 day and off the other. On the on day, stimulation parameters were adjusted to achieve maximal improvement in the motor portion of the Unified Parkinson's Disease Rating Scale (UPDRS items 19-31) ${ }^{15}$ without inducing dyskinesia. During PET imaging, the patients were in a stable on condition without medication for at least 12 hours before scanning. On the off day, all medications and stimulation were discontinued for at least 12 hours before imaging.

\section{Behavioral Tasks}

Because GPi DBS can affect the execution of simple movements and potentially also the learning of sequential movements, we assessed the effects of stimulation on each behavior separately. In each stimulation condition, the subjects performed two kinematically matched reaching tasks during PET imaging: (1) a motor sequence learning task (ML) and (2) a motor execution reference task (MR). The characteristics of these tasks have been described in detail previously., ${ }^{41,12}$ In both tasks, subjects moved a cursor on a digitizing tablet with their right hand. Movements were out and back from a central starting position to one of eight radial targets displayed on a computer screen. Targets appeared in synchrony with a 160-millisecond tone at a set

\begin{tabular}{|c|c|c|c|c|c|c|c|c|c|c|c|c|c|}
\hline \multirow{3}{*}{$\begin{array}{l}\text { Patient } \\
\text { No. }\end{array}$} & \multirow{3}{*}{$\begin{array}{l}\text { Age } \\
(\mathrm{yr})\end{array}$} & \multirow[b]{3}{*}{ Gender } & \multirow[b]{3}{*}{$\mathrm{H} \& \mathrm{Y}$} & \multirow[b]{3}{*}{ Medication $^{\mathrm{a}}$} & \multicolumn{9}{|c|}{ Stimulation Parameters } \\
\hline & & & & & \multirow{2}{*}{$\begin{array}{c}\text { Stimulation } \\
\text { Side }\end{array}$} & \multicolumn{2}{|c|}{ Contact $^{\mathrm{b}}$} & \multicolumn{2}{|c|}{$\begin{array}{l}\text { Pulse } \\
\text { Width } \\
\text { ( } \mu \text { sec) }\end{array}$} & \multicolumn{2}{|c|}{$\begin{array}{c}\text { Frequency } \\
(\mathrm{Hz})\end{array}$} & \multicolumn{2}{|c|}{$\begin{array}{l}\text { Amplitude } \\
\text { (V) }\end{array}$} \\
\hline & & & & & & $\mathrm{L}$ & $\mathrm{R}$ & $\mathrm{L}$ & $\mathrm{R}$ & $\mathrm{L}$ & $\mathrm{R}$ & $\mathrm{L}$ & $\mathrm{R}$ \\
\hline 1 & 32 & $\mathrm{~F}$ & 2 & 1,4 & $\mathrm{~L}$ & $3 / 0$ & - & 180 & - & 185 & - & 2.9 & - \\
\hline 2 & 55 & $\mathrm{~F}$ & 4 & 1,2 & $\mathrm{~L}$ & $2 / 1,3$ & - & 90 & - & 100 & - & 2.9 & - \\
\hline 3 & 51 & M & 4 & 1,3 & B & $1 / 3$ & $0,3 /$ case & 120 & 120 & 80 & 80 & 3.6 & 1.3 \\
\hline 4 & 42 & $\mathrm{~F}$ & 3 & 1,2 & B & $3 /$ case & $1 / 0$ & 180 & 180 & 70 & 100 & 1.6 & 2.6 \\
\hline 5 & 52 & M & 2 & 1,2 & B & $2 /$ case & 2/Case & 120 & 120 & 185 & 185 & 2.8 & 4.6 \\
\hline 6 & 70 & $\mathrm{M}$ & 4 & 1,2 & B & $2 /$ case & 2/Case & 90 & 90 & 130 & 130 & 3.0 & 3.0 \\
\hline 7 & 61 & M & 3 & 1,2 & B & $0 / 3$ & $0 / 2$ & 150 & 150 & 185 & 185 & 4.3 & 3.6 \\
\hline
\end{tabular}

${ }^{\mathrm{a}} 1$ = $\mathrm{L}$-dopa/carbidopa; $2=$ dopamine agonist; $3=$ anticholinergics; $4=$ monoamine oxidase inhibitors.

${ }^{\mathrm{b}} \mathrm{Numbers}$ indicating the position of the quadripolar contact(s) that were stimulated $(3=$ upper; $0=$ lower; interelectrode distance $=1.5 \mathrm{~mm})$ cathode/anode.

$\mathrm{H} \& \mathrm{Y}=$ off stimulation Hoehn and Yahr score at the 12-hour medication washout; $\mathrm{L}=$ left; $\mathrm{R}=$ right; $\mathrm{B}=$ bilateral. 
intertone interval. Subjects were instructed to reach for each target from the starting point and to synchronize the reversal of their movements with the tone. ${ }^{10}$ In the ML task, the eight targets appeared in a pseudorandom repeating order without repeating elements. ${ }^{12}$ The subjects were instructed to discover and learn the sequence order to anticipate the target and reach it as it appeared. At the end of each block trial, subjects were asked to indicate the order of the sequence verbally. During training sessions conducted before imaging, each subject experienced two or three different sequences; during PET scanning, entirely different sequences were used. In the MR task, targets appeared in a predictable counterclockwise order. ${ }^{10,11}$ To reach the target in synchrony with the tone, subjects had to initiate movement before it appeared within a time window of 250 milliseconds around each tone. All trial blocks lasted $90 \mathrm{sec}-$ onds.

In each subject, experimental task parameters were held constant across stimulation conditions. ${ }^{4}$ This was achieved by determining the shortest tone interval and the maximum target extent at which each patient could perform the MR task with $90 \%$ of hits in the off condition after the training session on day 1 . These parameters were used in both of the subsequent PET sessions (days 2 and 3). Tone intervals varied between 1 and 1.7 seconds (mean, $1.5 \pm 0.2 \mathrm{sec}-$ onds). Target extents varied between 0.4 and $4.8 \mathrm{~cm}$ (mean, $1.5 \pm 1.6 \mathrm{~cm}$ ); gains were adjusted so that the cursor movement on the screen was equivalent across subjects and conditions.

\section{Learning Performance: Behavioral Measures}

A computer controlled the experiment, generated screen displays, and acquired kinematic data from the digitizing tablet at $200 \mathrm{~Hz}$ as described previously. ${ }^{10,11}$ Because subjects were instructed to identify the sequence explicitly and to reach for the correct target before it appeared, anticipatory movements to the correct target were considered to reflect explicit learning. (Movements were considered anticipatory according to reaction time criteria defined previously. ${ }^{12}$ Correct responses were defined as movements with directional error of 22 degrees or less at peak velocity.) In each scan, learning performance was quantified by the total number of correctly anticipated movements during the 90 seconds of PET imaging. This psychophysical measure was termed the global learning index. Because of betweensubject variation in movement rate, we normalized this performance measure by the number of cycles completed during the PET epoch. We also obtained a declarative score defined as the number of accurate target locations reported by the patient at the end of each trial block $(0=$ unawareness of a repeating sequence to $8=$ complete correct sequence). Changes in the learning measures with stimulation were assessed by comparing on and off values using paired Student's $t$ tests. Changes were considered significant for $p$ values less than 0.05 , two-tailed.

\section{Positron Emission Tomography}

Patients were scanned on consecutive days in the on and off stimulation conditions. They fasted overnight before both imaging sessions. PET studies were performed in three-dimensional mode using the GE Advance Tomograph at North Shore University Hospital, Manhasset, NY ${ }^{16}$ according to the DBS imaging protocol that we described previously. ${ }^{4}$ In each of the two PET sessions (on and off), subjects were scanned while performing the ML and MR tasks in randomized order. All subjects performed the two tasks twice in each stimulation condition, with the exception of GPi DBS Patient 7, who was scanned only once during each task in both stimulator conditions. Psychophysical recording of learning performance was acquired with every run with the exception of GPi DBS Patients 3 and 6, in whom behavioral data were acquired only during a single PET epoch. Motor tasks were performed with the dominant right arm and an intravenous catheter was placed in the left arm for administration of $\mathrm{H}_{2}{ }^{15} \mathrm{O}$. Relative regional cerebral blood flow (rCBF) was estimated using a modification of the slow bolus method. ${ }^{4,12,17}$ Ethical permission for these studies was obtained from the institutional review board of North Shore University Hospital. Written consent was obtained from each subject after they were given detailed explanation of the procedures.

\section{Effects of Stimulation on Brain Activation during Learning}

We sought to identify brain regions in which GPi stimulation significantly altered rCBF during motor sequence learning. This was achieved with SPM 99 software (Wellcome Department of Cognitive Neurology, London, UK) using a two-factor analysis of variance that included all four conditions $\left(\mathrm{ML}_{\mathrm{ON}}, \mathrm{MR}_{\mathrm{ON}}, \mathrm{ML}_{\mathrm{OFF}}, \mathrm{MR}_{\mathrm{OFF}}\right){ }^{4}$ In this way, we assessed stimulation effects on activation during learning as well as potential interaction effects with motor execution. All scans were entered simultaneously in the design matrix, and the differences were detected by specifying a contrast of (1, $-1,-1,1)$. We hypothesized that, during learning, stimulation will alter rCBF within the set of voxels known through previous $\mathrm{H}_{2}{ }^{15} \mathrm{O} / \mathrm{PET}$ studies to be specifically activated by the ML task. To confine statistical analysis to this known set of voxels, we created a mask ${ }^{4}$ defined by ML-MR $\mathrm{rCBF}$ differences obtained in an independent population comprising 22 unmedicated PD patients and 18 normal volunteers who performed both tasks. ${ }^{12}$ This mask was compiled with 73 pre-existing learning and reference scan pairs, and a threshold was set at $p$ value less than 0.001 . The mask (Fig 1) sampled bilateral learning-related $\mathrm{rCBF}$ increases in the DLPFC, PMC, pre-supplementary motor area, precuneus, and posterior parietal cortical regions. Stimulation effects on learning activation within the population mask were considered to be hypothesis driven and significant for $p$ values less than 0.01, uncorrected for independent multiple comparisons. Stimulation effects outside this mask were considered to be hypothesis generating for $p$ values less than 0.001 , uncorrected for multiple comparisons, and significant if they survived a correction for multiple comparisons at $p$ values equaling 0.05 . Coordinates were reported in the standard anatomical space developed at the Montreal Neurological Institute. ${ }^{18}$

In addition, we determined whether the effects of stimulation at each significant voxel were specific for learning, or 


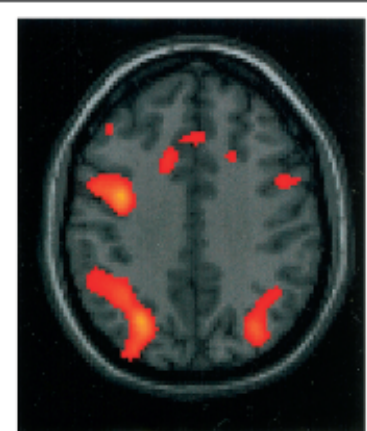

$(\mathrm{z}=38 \mathrm{~mm})$

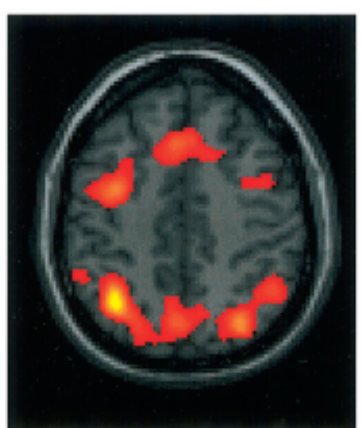

$(\mathrm{z}=46 \mathrm{~mm})$

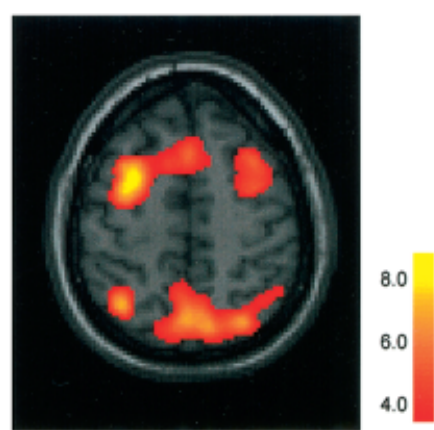

$(\mathrm{z}=54 \mathrm{~mm})$

Fig 1. Motor sequence learning: activation mask. Brain activation during the motor sequence learning task in a pre-existing population of normal subjects and untreated Parkinson's disease (PD) patients (see text). The task activated the dorsolateral prefrontal cortex (left), the premotor cortex and the rostral supplementary motor area (pre-supplementary motor area, right), and the precuneus and posterior parietal cortical regions (right and middle). The activations were thresholded at $\mathrm{Z}=3.21$ ( $\mathrm{p}<0.001$ ) and utilized as a mask for hypothesis testing in the subsequent motor learning experiments performed to assess the effects of stimulation on brain activation.

whether they were confounded by the effect of treatment on motor execution. This was achieved by post hoc testing to assess changes in MR activation in the voxels that exhibited significant effects of intervention during ML task performance. rCBF changes at these voxels were considered to relate to motor execution if the on-off differences in rCBF during MR performance were significant for $p$ values less than 0.05 (paired Student's $t$ test, two-tailed).

\section{Results}

Effects of Stimulation on Learning Performance

UPDRS motor ratings and measures of learning performance in the off and on states are presented for each patient in Table 2. GPi stimulation improved UPDRS motor ratings off medication (from $41.9 \pm 17.3$ $[$ mean $\pm \mathrm{SD}]$ to $25.3 \pm 11.1$ [37.0\%], $p<0.01)$, as well as kinematic parameters during the MR task as reported previously. ${ }^{4}$ Movement time during the ML task also declined with stimulation (from $609.2 \pm$ 127.4 to $539.8 \pm 142.8$ milliseconds [11.4\%], $p<$ 0.03). During this task, GPi stimulation resulted in significant improvement in both the global learning in$\operatorname{dex}(p<0.01)$ and in declarative score $(p<0.001$; see Table 2; Fig 2). Changes in learning parameters with stimulation did not correlate $\left(R^{2}<0.15\right)$ with concurrent reductions in either movement time or UPDRS motor ratings.

\section{Effects of Stimulation on Brain Activation during Learning}

The main effect of learning (ML-MR) was topographically similar in both the off and on conditions. The map of learning-related responses in this cohort of advanced PD patients was consistent with the spatial distribution described previously in normal subjects and

Table 2. Clinical Ratings and Performance Indices

\begin{tabular}{|c|c|c|c|c|c|c|}
\hline \multirow{2}{*}{$\begin{array}{l}\text { Patient } \\
\text { No. }\end{array}$} & \multicolumn{2}{|c|}{ UPDRS Ratings $^{\mathrm{a}}$} & \multicolumn{2}{|c|}{ Global Learning Index ${ }^{b}$} & \multicolumn{2}{|c|}{ Declarative Score $^{c}$} \\
\hline & OFF & $\mathrm{ON}$ & OFF & $\mathrm{ON}$ & OFF & $\mathrm{ON}$ \\
\hline 1 & 40 & 23 & 3.88 & 4.75 & 6 & 8 \\
\hline 2 & 30 & 21 & 1.56 & 2.56 & 3 & 6 \\
\hline 3 & 42 & 30 & 2.09 & 3.55 & 6 & 8 \\
\hline 4 & 73 & 43 & 0.63 & 0.38 & 1 & 2 \\
\hline 5 & 50 & 34 & 2.06 & 4.56 & 6 & 8 \\
\hline 6 & 17 & 11 & 0.43 & 4.00 & 3 & 6 \\
\hline 7 & 41 & 11 & 1.43 & 6.00 & 6 & 8 \\
\hline Average & 41.9 & 25.3 & 1.84 & 3.46 & 4.27 & 6.36 \\
\hline
\end{tabular}

${ }^{a}$ Motor ratings in the off medication state; OFF and ON refer to stimulation condition (see text).

${ }^{b}$ Mean number of correctly anticipated movements per cycle (see text).

${ }^{\mathrm{c}}$ Number of correctly reported target locations averaged across trial blocks (see text).

UPDRS = Unified Parkinson's Disease Rating Scale. 

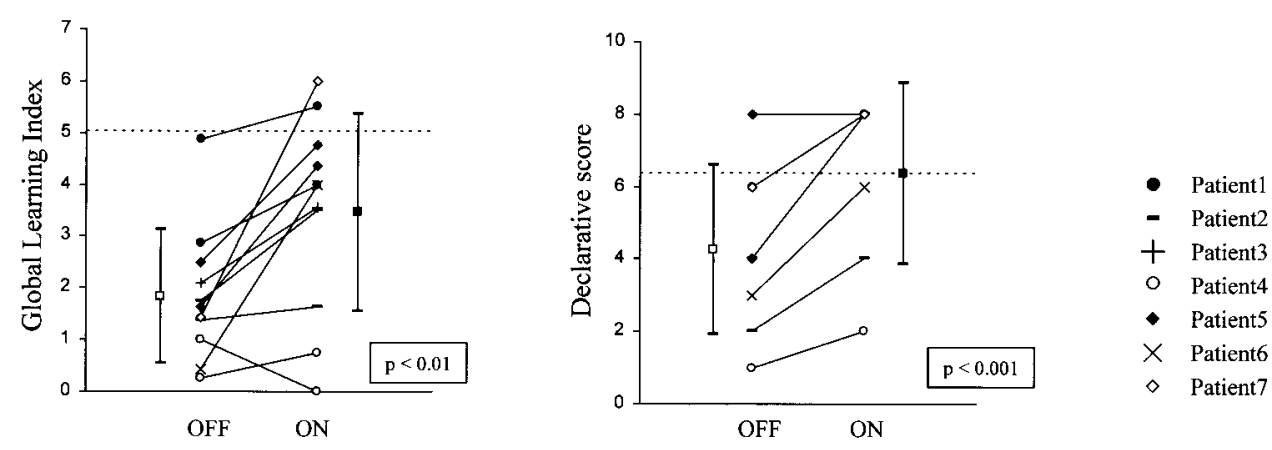

Fig 2. Effects of deep brain stimulation of the internal globus pallidus on sequence learning performance (see text). This intervention significantly improved both the global learning index (left) and declarative scores (right). A key for symbols representing individual patients (see Table 1) is at the right. Separate lines connect ON and OFF values for the first and second trials conducted in each session (see text). The dotted lines represent the mean values for normal subjects. ${ }^{12}$

mild, unmedicated PD patients. ${ }^{12}$ Conforming with the independent population mask (see Fig 1), we localized task-related activations bilaterally to the DLPFC, PMC, pre-supplementary motor area, and parietal cortex.

Areas in which DBS significantly enhanced learningspecific activation responses $\left([\mathrm{ML}-\mathrm{MR}]_{\mathrm{ON}}>\right.$ $\left.[\mathrm{ML}-\mathrm{MR}]_{\mathrm{OFF}}\right)$ are presented in Figure 3 and Table 3. Hypothesis-driven searches within the ML-MR population mask showed significant stimulationinduced augmentation of activation in the left DLPFC Brodmann Area (BA 9), and bilaterally in the PMC (BA 6) and in posterior parietal and occipital association areas (left BA 7/40, right BA 19). Stimulation did not cause significant $\mathrm{rCBF}$ decrements during learning. In these regions, stimulation did not have a significant effect on blood flow during MR task performance (see Fig 3, bar graphs; see Table 3). Outside the mask, regional changes in brain activation during learning did not reach the threshold for hypothesis generation ( $p=$ 0.001 , uncorrected). Note, however, that two additional areas were identified at the more lenient uncorrected threshold of $p$ values equaling 0.01 . These stimulation-mediated changes were localized to the right prefrontal cortex (BA 9; $x=40 ; y=6 ; z=24)$ and the left parahippocampal gyrus (BA 27; $x=-22$, $y=-30, z=-8)$.

\section{Discussion}

Effects of Stimulation on Motor Sequence Learning A salient behavioral result of our study is the finding of improved sequence learning performance during GPi stimulation. This observation generally is consistent with neuropsychological studies demonstrating an enhancement of certain aspects of executive functioning with DBS for parkinsonism. ${ }^{8,9}$ Motor sequence learning as assessed using the ML and MR tasks is abnormal in early-stage PD patients in the unmedicated state. ${ }^{12}$ It is likely that stimulation-mediated improvements in this cognitive process are subserved by mechanisms similar to the observed neuropsychological changes. Nonetheless, having restricted our study to the learning of sequences, we cannot exclude the possibility that stimulation could result in cognitive decrements in other domains such as verbal fluency and visuoconstructional function. ${ }^{6}$

The increase in learning scores with GPi stimulation

Table 3. Brain Regions in which GPi DBS Significantly Altered Regional Activation during Motor Sequence Learning

\begin{tabular}{|c|c|c|c|c|c|c|c|c|}
\hline \multirow[b]{2}{*}{ Brain Region } & \multicolumn{3}{|c|}{ Coordinates $(\mathrm{mm})$} & \multirow[b]{2}{*}{$Z$ Score } & \multicolumn{4}{|c|}{ Mean Adjusted Cerebral Blood Flow $(\mathrm{ml} / \mathrm{min} / 100 \mathrm{gm})$} \\
\hline & $x$ & $y$ & $z$ & & $\mathrm{ML}_{\mathrm{OFF}}$ & $\mathrm{ML}_{\mathrm{ON}}$ & $\mathrm{MR}_{\mathrm{OFF}}$ & $\mathrm{MR}_{\mathrm{ON}}$ \\
\hline \multicolumn{9}{|l|}{ Increases } \\
\hline Left DLPFC (BA 9) & -38 & 34 & 32 & 3.05 & $75.9 \pm 5.8$ & $78.9 \pm 4.8$ & $76.1 \pm 5.9$ & $74.9 \pm 5.4$ \\
\hline \multirow[t]{2}{*}{ Right PMC (BA 6) } & 30 & -2 & 56 & 3.34 & $84.9 \pm 5.0$ & $87.9 \pm 4.5$ & $85.4 \pm 4.0$ & $83.1 \pm 5.2$ \\
\hline & 26 & 16 & 56 & 3.19 & $76.3 \pm 7.9$ & $79.8 \pm 7.8$ & $76.0 \pm 5.9$ & $75.2 \pm 5.8$ \\
\hline Left PMC (BA 6) & -26 & -2 & 62 & 2.60 & $84.9 \pm 8.1$ & $88.4 \pm 8.6$ & $86.2 \pm 5.6$ & $85.7 \pm 8.1$ \\
\hline Left posterior parietal (BA 7/40) & -36 & -52 & 54 & 2.68 & $83.5 \pm 9.5$ & $85.8 \pm 8.2$ & $84.4 \pm 9.6$ & $82.6 \pm 8.3$ \\
\hline Right occipital (BA 19) & 40 & -82 & 32 & 3.15 & $67.5 \pm 3.4$ & $69.6 \pm 4.2$ & $67.9 \pm 3.3$ & $66.4 \pm 3.7$ \\
\hline
\end{tabular}

GPi DBS = deep brain stimulation of the internal globus pallidus; $\mathrm{ML}=$ motor sequence learning task; $\mathrm{MR}=$ motor execution reference task; $\mathrm{DLPFC}=$ dorsolateral prefrontal cortex; PMC $=$ premotor cortex. 


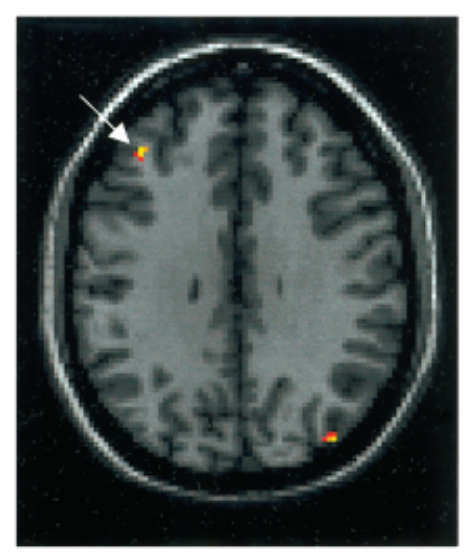

Left DLPFC $(x=-38, y=34, z=32)$
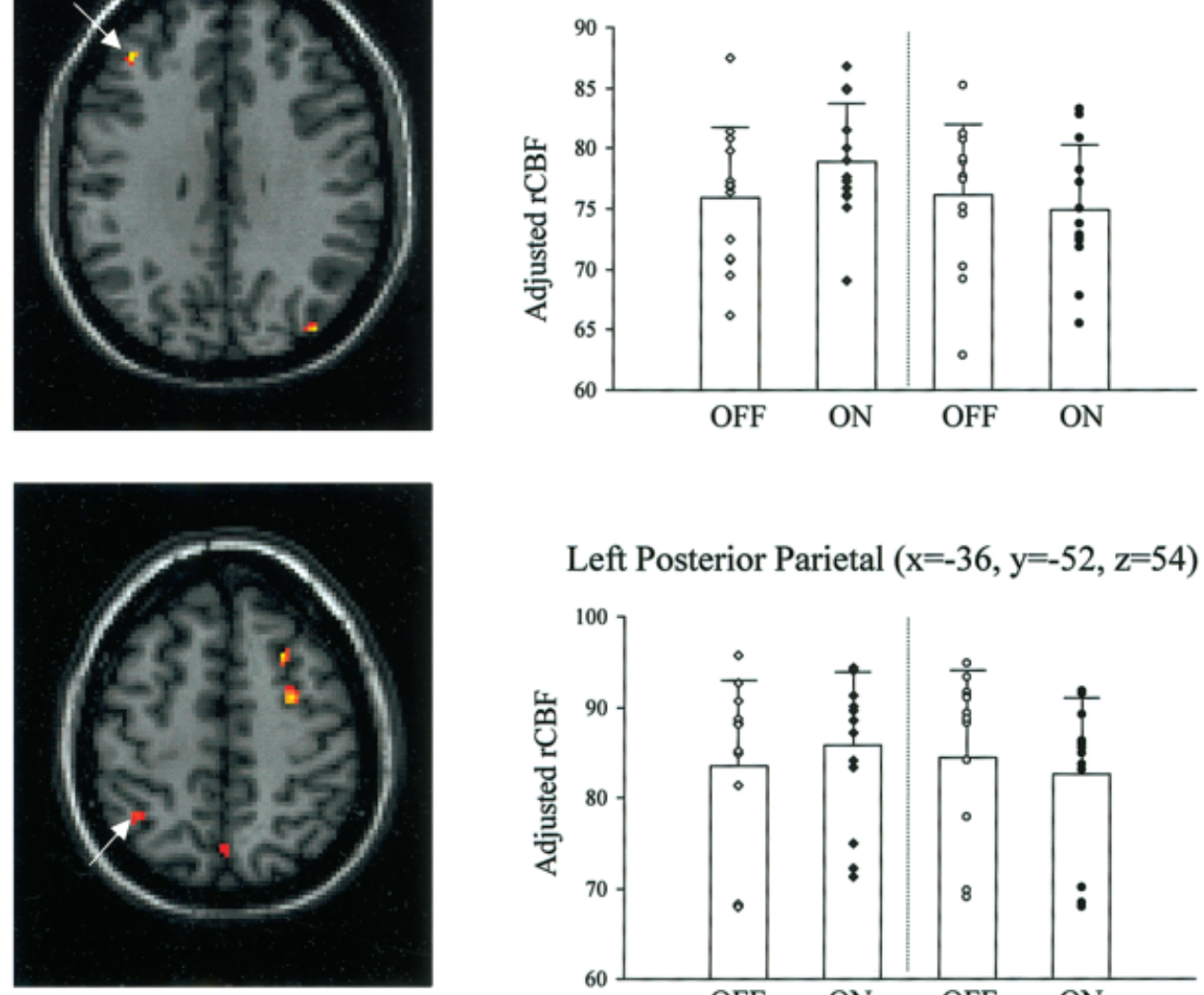

\section{Left Posterior Parietal $(x=-36, y=-52, z=54)$}
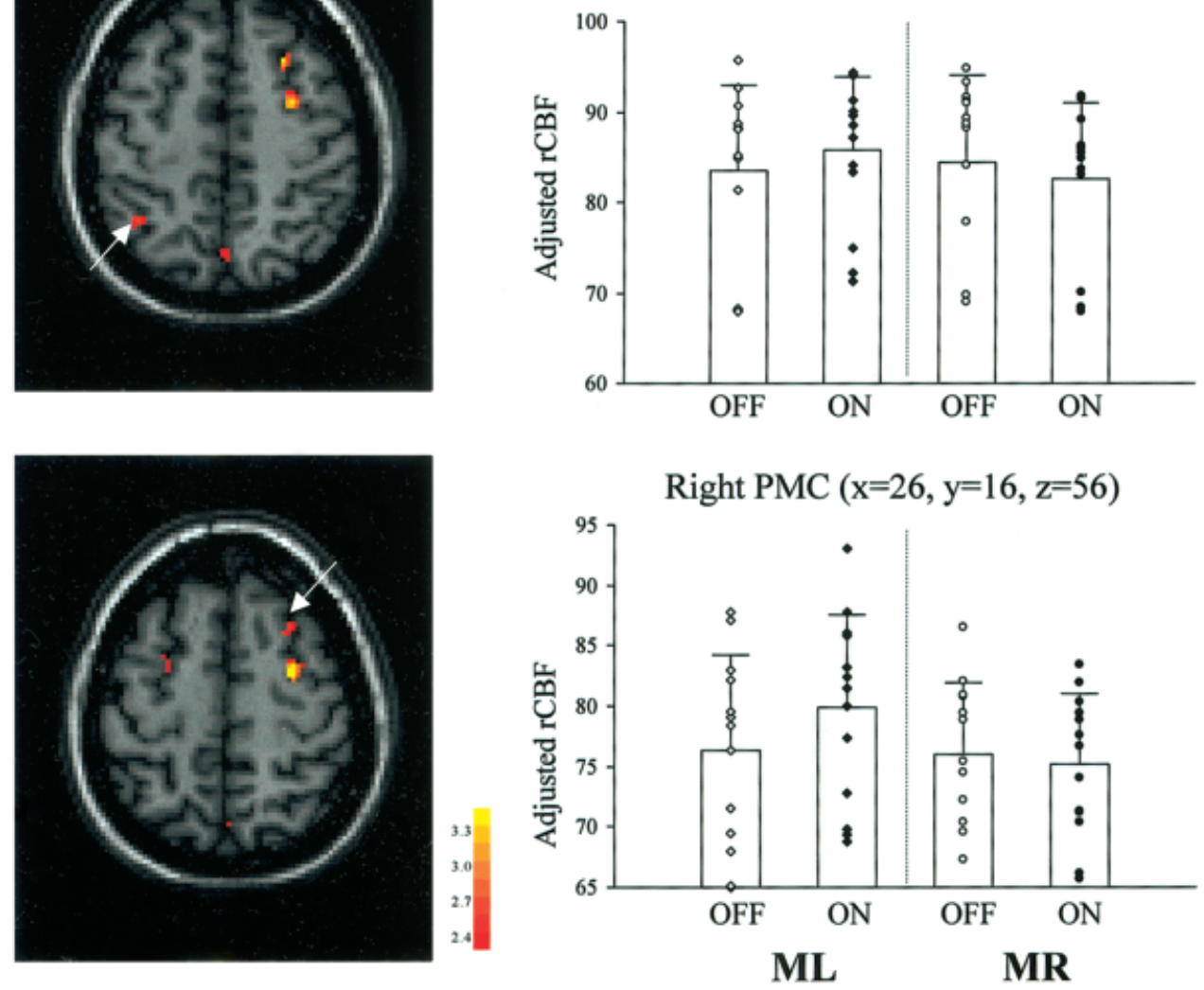

Fig 3. Brain regions in which globus pallidus deep brain stimulation (GPi DBS) significantly altered regional blood flow ( $r C B F$ ) during motor sequence learning. Bar graphs of $r C B F$ measured during the motor learning $(M L)$ task and the motor execution reference $(M R)$ task are at the right of each slice. GPi DBS increased rCBF during learning in the dorsolateral prefrontal cortex (DLPFC, top), the posterior parietal cortex (middle), and the premotor cortex (PMC, bottom). In these brain regions, there were no significant effects of stimulation on $r C B F$ measured during the motor execution reference task (see text). The color stripe represents $\mathrm{Z}$-scores thresholded at 2.56; $\mathrm{p}<0.01$. Standard deviations are represented by error bars.

is not likely to have resulted from concurrent improvement in motor performance. First, the changes in learning parameters with stimulation did not correlate with reductions in either movement time or UPDRS motor ratings. Second, DBS improved sequence learning performance as measured by the number of correct anticipatory movements or by verbal report. In our prior study of normal subjects and patients with early- 
stage $\mathrm{PD}$, we found these measures to be highly correlated. ${ }^{12}$ Similar significant correlations $\left(R^{2}, \approx 0.70 \%\right.$; $p<0.001)$ between the two learning parameters were found in this cohort of more severely affected patients, both on and off stimulation. Although a disparity between these measures would be expected in the presence of significant akinesia, this is not the case in this study. Last, the significant treatment-mediated changes in brain activation that occurred during sequence learning were topographically specific for this task: stimulation did not affect neural activity in these regions during simple motor execution.

We recently have reported reduced sequence learning performance during intravenous L-dopa infusion..$^{13}$ This observation is compatible with prior cognitive studies utilizing highly demanding executive tasks, especially in patients with more advanced symptoms. ${ }^{19-22}$ Despite the less severe motor involvement of our L-dopa cohort, this intervention tended to impair learning performance. In contrast, GPi stimulation improved this aspect of cognitive functioning in a relatively more affected patient cohort.

\section{Effects of Stimulation on Brain Activation during Learning}

GPi DBS caused increases in neural activation in DLPFC, PMC, posterior parietal cortex, and the occipital association area. These areas are known to be involved in the normal learning of new movement sequences. $^{23-27}$ The role of the DLPFC in the early phases of motor sequence learning is widely accepted; albeit hemispheric functional asymmetry in this region may vary according to experimental design. ${ }^{12,23,24,26,27}$ Our finding of stimulation-mediated increases in neural activity in this region is compatible with prior human studies indicating changes in blood flow and metabolism in DLPFC after pallidotomy. ${ }^{28,29}$ Indeed, in primates the rostrodorsal and dorsomedial portions of GPi project to the prefrontal cortex (BA 9 and BA 46, respectively) via the ventral thalamus. ${ }^{30}$ Although in our patients stimulation parameters were adjusted to maximize motor benefit, the stimulation effects are likely to extend to the more dorsally situated output channels that modulate prefrontal learning circuits. Indeed, the more dorsal cathode contacts (2 or 3 ) were active in five of the seven GPi DBS patients who composed our cohort (see Table 1).

The PMC contains numerous sequence-specific neurons, especially in its dorsorostral subdivision. ${ }^{31,32} \mathrm{Sev}-$ eral PET studies of explicit motor sequence learning have showed PMC activation, most pronounced on the right. ${ }^{12,24,26,33}$ The GPi neurons that project via the ventrolateral thalamic nucleus to the ventral PMC also are located ventrolaterally ${ }^{30}$. During motor sequence learning, GPi stimulation may directly exert significant local effects on the ventrolateral pallidum and influence the PMC through relays in the ventrolateral thalamic nucleus. This pathway may be necessary to optimize externally guided movements during the initial phases of motor sequence learning.

GPi DBS also can increase learning-related activation in cortical areas that are not elements of the higher-order cortico-striato-pallidothalamic circuits. Specifically, this intervention enhanced neural activity in the posterior parietal cortex (BA 7/40) and the occipital association area (BA 19). Prior PET studies have demonstrated that these regions are activated during motor sequence learning. ${ }^{12,24-26,33}$ Specifically, posterior parietal activation has been associated with the later phases of sequence learning. ${ }^{27}$ Although pallidal stimulation does not directly influence posterior cortical regions, ${ }^{14,34}$ it is likely that these $\mathrm{rCBF}$ changes occurred indirectly via the DLPFC or PMC through alterations in the activity of transcortical pathways. ${ }^{12,35}$

We note that the regions functionally altered by GPi stimulation were rather small anatomically. However, the validity of these effects is supported by their localization within an independent population mask obtained from many subjects who performed the same pair of kinematically controlled tasks. Moreover, these specific areas represented the major nodes of a specific functional covariance pattern ${ }^{14}$ that was significantly modulated by stimulation. ${ }^{36}$ Last, ancillary image analysis using a traditional region of interest approach showed significant $(p<0.05)$ or near significant $(p<$ 0.1 ) changes in $\mathrm{rCBF}$ in the larger but architectonically heterogeneous brain volumes that surrounded the significant voxel clusters.

The predominant effects of stimulation on brain activation during motor sequence learning occurred within the hypothesis-testing mask. Effects within these areas were considered to be part of the distributed network of regions activated during learning and thus were not independent of each other. ${ }^{4,14}$ For this reason, we chose a relatively liberal threshold for significance within the mask, without a correction for multiple comparisons. In contrast, this consideration may not apply outside the mask where there is no empiric basis for hypothesis testing. Although no region outside the mask survived a more stringent criterion for significance, two potentially relevant areas were identified at a liberal threshold. Within the mask, a prominent stimulation effect was localized to the left DLPFC. A change of comparable magnitude was present just outside the mask in the right prefrontal cortex (BA 9). This finding is consistent with the bilateral role of this region in motor task performance, especially in parkinsonian subjects. ${ }^{12}$ We also identified a treatment effect during learning in the left parahippocampal gyrus. Although this region had 
not been found to be activated in our simple serial reaction time learning task, increased hippocampal activation had been reported in PD patients performing a more demanding problem solving paradigm. ${ }^{37}$ It is not known whether DBS can improve performance and/or alter hippocampal functioning during this planning task.

The precise mechanism by which GPi DBS improves the learning of sequential information is not understood. The effects of STN DBS on this cognitive process are also unknown. PET data acquired during simple joystick movement suggest that stimulation at this site causes relatively greater increases in DLPFC activity, ${ }^{3}$ which might also influence learning performance. Focal stereotaxic interventions in the pallidum or STN do not inherently interfere with the functioning of cortico-cortical pathways. Therefore, assuming that the individual cortical nodes of these functional networks remain structurally intact, a surgical intervention at a remote site in the basal ganglia may facilitate brain activation and sequence learning, perhaps by reducing noisy pallidal output. ${ }^{38}$ In contrast, less specific treatments such as L-dopa can improve motor signs but also may impair aspects of cognitive functioning. ${ }^{13,19-23}$ Indeed, pharmacological alteration of the function of the DLPFC and its efferent projections may interfere with the normal rostrocaudal transfer of information that is fundamental to the explicit learning process. ${ }^{27,39}$ Further behavioral and imaging studies of subjects undergoing stimulation and dopaminergic therapy, alone and in combination, will be needed to compare the effects of these interventions on cognitive functioning in parkinsonism.

This research was supported by the National Institutes of Health (NIH RO1 NS 35069, D.E.; NIH KO8 NS 01961, M.F.G.; NIH K23 NS 02204, M.J.M.; and NIH K24 NS 02101, D.E.), the National Parkinson Foundation (D.E.), Veola T. Kerr Fellowship of the Parkinson Disease Foundation (M.F.), and by the Cotzias Fellowship of the American Parkinson Disease Association (D.E.).

We thank Drs William Koller, Kelly Lyons, and John Hammerstad for patient referral, Dr Thomas Chaly for radiochemistry support, and Christine Edwards for editorial assistance. We acknowledge the valuable technical support provided by Dr Abdel Belakhleff and Claude Margouleff.

\section{References}

1. Starr PA, Vitek JL, Bakay RA. Ablative surgery and deep brain stimulation for Parkinson's disease. Neurosurgery 1998;43: 989-1013.

2. Limousin P, Krack P, Pollak P, et al. Electrical stimulation of the subthalamic nucleus in advanced Parkinson's disease. N Engl J Med 1998;339:1105-1111.
3. Limousin P, Greene J, Pollak P, et al. Changes in cerebral activity pattern due to subthalamic nucleus or internal pallidum stimulation in Parkinson's disease. Ann Neurol 1997;42: 283-291.

4. Fukuda M, Mentis MJ, Ghilardi MF, et al. Functional correlates of pallidal stimulation for Parkinson's disease. Ann Neurol 2001;49:155-165.

5. Wichmann T, DeLong MR. Functional and pathophysiological models of the basal ganglia. Curr Opin Neurobiol 1996;6: 751-758.

6. Troster AI, Fields JA, Wilkinson SB, et al. Unilateral pallidal stimulation for Parkinson's disease: neurobehavioral functioning before and 3 months after electrode implantation. Neurology 1997;49:1078-1083.

7. Vingerhoets G, Van der Linden C, Lannoo E, et al. Cognitive outcome after unilateral pallidal stimulation in Parkinson's disease. J Neurol Neursurg Psychiatry 1999;66:297-304.

8. Ardouin C, Pillon B, Peiffer E, et al. Bilateral subthalamic or pallidal stimulation for Parkinson's disease affects neither memory nor executive functions: a consecutive series of 62 patients. Ann Neurol 1999;46:217-223.

9. Jahanshahi M, Ardouin CM, Brown RG, et al. The impact of deep brain stimulation on executive function in Parkinson's disease. Brain 2000;123:1142-1154.

10. Ghez CP, Favilla M, Ghilardi MF, et al. Discrete and continuous planning of hand movements and isometric force trajectories. Exp Brain Res 1997;115:217-233.

11. Ghilardi M, Ghez C, Moeller J, et al. Patterns of regional brain activation associated with different aspects of motor learning. Brain Res 2000;871:127-145.

12. Nakamura T, Ghilardi MF, Mentis M, et al. Functional networks in motor sequence learning: abnormal topographies in Parkinson's disease. Hum Brain Mapp 2001;12:42-60.

13. Ghilardi MF, Ghez CP, Feigin A, et al. Motor sequence learning in Parkinson's disease: differential effects of levodopa and DBS. Neurology 2001;56:A147.

14. Fukuda M, Mentis MJ, Ma Y, et al. Networks mediating the clinical effects of pallidal brain stimulation for Parkinson's disease: a PET study of resting state glucose metabolism. Brain 2001;124:1601-1609.

15. Fahn S, Elton R. Unified Parkinson's disease rating scale. In: Fahn S, Marsden C, Calne D, Goldstein M, eds. Recent developments in Parkinson's disease. Vol 2. New York: Macmillan, 1987:293-304.

16. Dhawan V, Kazumata K, Robeson W, et al. Quantitative brain PET: comparison of $2 \mathrm{D}$ and $3 \mathrm{D}$ acquisition on the GE Advance Scanner. Clin Positron Imaging 1998;1:135-144.

17. Silbersweig DA, Stern E, Frith CD, et al. Detection of thirtysecond cognitive activations in single subjects with positron emission tomography: a new low-dose $\mathrm{H} 2(15) \mathrm{O}$ regional cerebral blood flow three-dimensional imaging technique. J Cereb Blood Flow Metab 1993;13:617-629.

18. Collins L, Neelin P, Peters T, Evans A. Automatic 3-D intersubject registration of MR volumetric data in standardized Talairach space. J Comput Assist Tomogr 1994;18:192205.

19. Gotham AM, Brown RG, Marsden CD. "Frontal" cognitive function in patients with Parkinson's disease "on" and "off" levodopa. Brain 1988;111:299-321.

20. Poewe W, Berger W, Benke T, Schelosky L. High-speed memory scanning in Parkinson's disease: adverse effects of levodopa. Ann Neurol 1991;29:670-673.

21. Kulisevsky J, Avila A, Barbanoj M, et al. Acute effects of levodopa on neuropsychological performance in stable and fluctuating Parkinson's disease patients at different levodopa plasma levels. Brain 1996;119:2121-2132. 
22. Cools R, Barker RA, Sahakian BJ, Robbins TW. Enhanced or impaired cognitive function in Parkinson's disease as a function of dopaminergic medication and task demands. Cereb Cortex 2001;11:1136-1143.

23. Tulving E, Kapur S, Craik FIM, et al. Hemispheric encoding/ retrieval asymmetry in episodic memory: positron emission tomography findings. Proc Natl Acad Sci USA 1994;91: 2016-2020.

24. Grafton S, Hazeltine E, Ivry R. Functional mapping of sequence learning in normal humans. J Cogn Neurosci 1995;7: 497-510.

25. Rauch S, Savage C, Brown H, et al. A PET investigation of implicit and explicit sequence learning. Hum Brain Mapp 1995;3:271-286.

26. Honda M, Deiber M-P, Ibanez V, et al. Dynamic cortical involvement in implicit and explicit motor learning: a PET study. Brain 1998;121:2159-2173.

27. Sakai K, Hikosaka O, Miyauchi S, et al. Transition of brain activation from frontal to parietal areas in visuomotor sequence learning. J Neurosci 1998;18:1827-1840.

28. Eidelberg D, Moeller J, Ishikawa T, et al. Regional metabolic correlates of surgical outcome following unilateral pallidotomy for Parkinson's disease. Ann Neurol 1996;39:450-459.

29. Samuel M, Ceballos-Baumann AO, Turjanski N, et al. Pallidotomy in Parkinson's disease increases supplementary motor area and prefrontal activation during performance of volitional movements an H2(15)O PET study. Brain 1997;120: 1301-1313.

30. Middleton FA, Strick PL. Basal ganglia and cerebellar loops: motor and cognitive circuits. Brain Res Brain Res Rev 2000; $31: 236-250$.
31. Mushiake H, Inase M, Tanji J. Neuronal activity in the primate premotor, supplementary, and precentral motor cortex during visually guided and internally determined sequential movements. J Neurophysiol 1991;66:705-718.

32. Wise SP, Boussaoud D, Johnson PB, Caminiti R. On the development of procedural knowledge. J Exp Psychol Learn Mem Cogn 1989;15:1047-1060.

33. Jenkins IH, Brooks DJ, Nixon PD, et al. Motor sequence learning: a study with positron emission tomography. J Neurosci $1994 ; 14: 3775-3790$.

34. Davis KD, Taub E, Houle S, et al. Globus pallidus stimulation activates the cortical motor system during alleviation of parkinsonian symptoms. Nat Med 1997;3:671-674.

35. Goldman-Rakic PS. Circuitry of primate prefrontal cortex and regulation of behavior by representational memory. In: Plum F, Mountcastle VB, eds. Handbook of physiology, section 1: the nervous system. Vol V. Higher functions of the brain. Part 1 . Bethesda: American Physiological Society, 1987:373-417.

36. Eidelberg D, Ghilardi MF, Fukuda M, et al. Effects of antiparkinsonian therapy on motor sequence learning: a PET study. Neurology 2001;56:A270.

37. Dagher A, Owen AM, Boecker H, Brooks DJ. The role of the striatum and hippocampus in planning. A PET activation study in Parkinson's disease. Brain 2001;124:1020-1032.

38. Brown P, Marsden CD. What do the basal ganglia do? Lancet 1998;351:1801-1804.

39. Matay VS, Tessitore A, Callicott JH, et al. Dopaminergic modulation of cortical function in patients with Parkinson's disease. Ann Neurol 2002;51:156-164. 